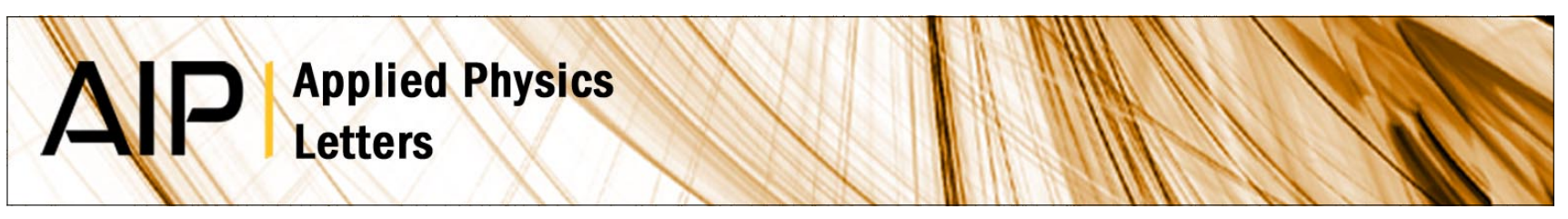

\title{
The effect of Bi composition to the optical quality of GaAs1xBix
}

A. R. Mohmad, F. Bastiman, C. J. Hunter, J. S. Ng, S. J. Sweeney et al.

Citation: Appl. Phys. Lett. 99, 042107 (2011); doi: 10.1063/1.3617461

View online: http://dx.doi.org/10.1063/1.3617461

View Table of Contents: http://apl.aip.org/resource/1/APPLAB/v99/i4

Published by the American Institute of Physics.

\section{Related Articles}

GaN directional couplers for integrated quantum photonics

Appl. Phys. Lett. 99, 161119 (2011)

Room temperature spin filtering effect in GaNAs: Role of hydrogen

Appl. Phys. Lett. 99, 152109 (2011)

Carrier localization and related photoluminescence in cubic AIGaN epilayers

J. Appl. Phys. 110, 063517 (2011)

Enhanced magnetization in erbium doped $\mathrm{GaN}$ thin films due to strain induced electric fields

Appl. Phys. Lett. 99, 122506 (2011)

Origin of Berreman effect in GaN layers on sapphire substrates

J. Appl. Phys. 110, 053519 (2011)

\section{Additional information on Appl. Phys. Lett.}

Journal Homepage: http://apl.aip.org/

Journal Information: http://apl.aip.org/about/about_the_journal

Top downloads: http://apl.aip.org/features/most_downloaded

Information for Authors: http://apl.aip.org/authors

\section{ADVERTISEMENT}

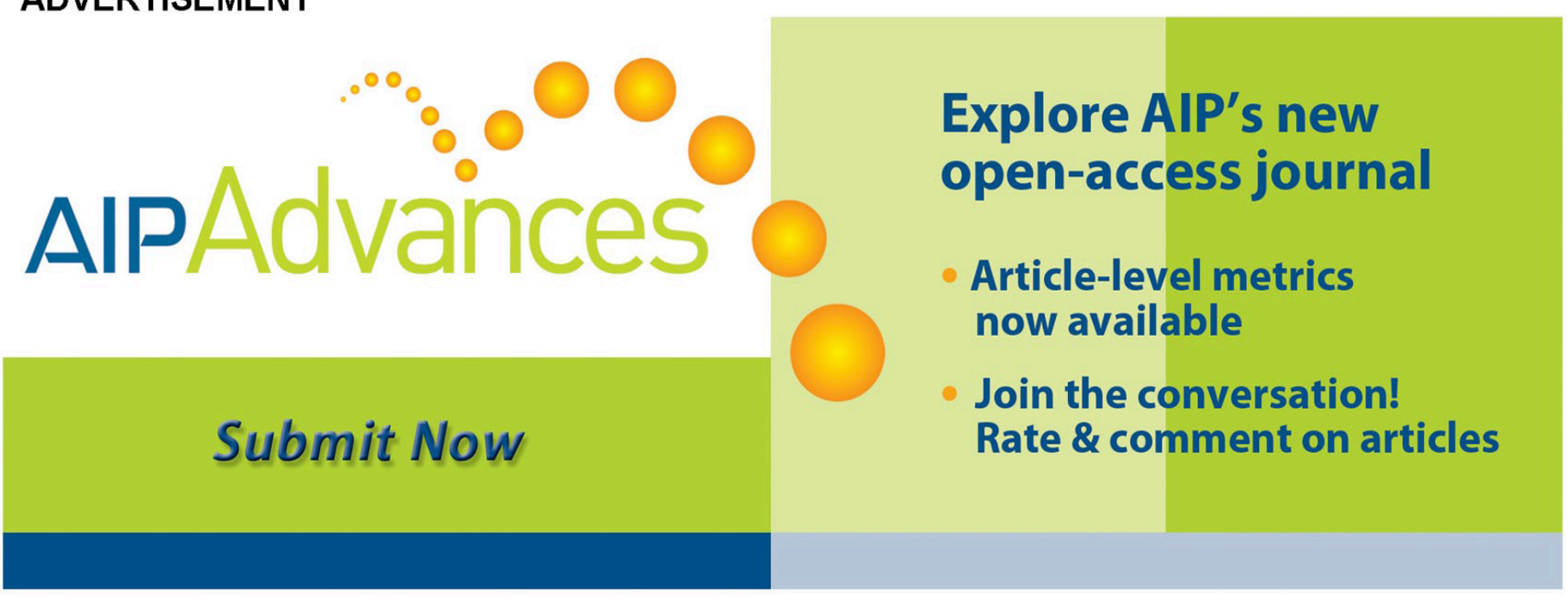




\title{
The effect of Bi composition to the optical quality of $\mathrm{GaAs}_{1-\mathbf{x}} \mathrm{Bi}_{\mathbf{x}}$
}

\author{
A. R. Mohmad, ${ }^{1,2}$ F. Bastiman, ${ }^{1}$ C. J. Hunter, ${ }^{1}$ J. S. Ng, ${ }^{1}$ S. J. Sweeney, ${ }^{3}$ \\ and J. P. R. David ${ }^{1, a)}$ \\ ${ }^{1}$ Department of Electronic and Electrical Engineering, University of Sheffield, Sir Frederick Mappin Building, \\ Mappin Street, Sheffield S1 3JD, United Kingdom \\ ${ }^{2}$ Institute of Microengineering and Nanoelectronics, National University of Malaysia (UKM), 43000 Bangi, \\ Selangor, Malaysia \\ ${ }^{3}$ Advanced Technology Institute and Department of Physics, University of Surrey, Guildford, Surrey GU2 7XH, \\ United Kingdom
}

(Received 21 June 2011; accepted 9 July 2011; published online 28 July 2011)

\begin{abstract}
$\mathrm{GaAs}_{1-\mathrm{x}} \mathrm{Bi}_{\mathrm{x}}$ alloys grown by molecular beam epitaxy for $x$ up to 0.06 were studied by photoluminescence (PL). The results indicate that dilute fractions of bismuth (Bi) with $x<0.025$ improve the material quality of this low temperature growth alloys by reducing the density of gallium (Ga) and/or arsenic related defects. The crystal quality starts to degrade at higher $\mathrm{Bi}$ concentration probably due to significant amount of Bi-related defects, $\mathrm{Bi}_{\mathrm{Ga}}$. However, the room temperature PL intensity continues to increase with $\mathrm{Bi}$ content for the range studied due to greater band-gap offset between GaAs and $\mathrm{GaAs}_{1-\mathrm{x}} \mathrm{Bi}_{\mathrm{x}}$. Analysis carried out shows no correlation between localization effects and the room temperature PL enhancement. (C) 2011 American Institute of Physics. [doi:10.1063/1.3617461]
\end{abstract}

Recently, bismuth (Bi) containing semiconductors have attracted increasing interest due to the large band gap reduction of $\sim 88 \mathrm{meV} / \% \mathrm{Bi}$ for $\mathrm{GaAs}_{1-\mathrm{x}} \mathrm{Bi}_{\mathrm{x}}$, relatively temperature insensitive band gap and large spin-orbit splitting. ${ }^{1-3}$ Hence, such alloys are promising for long wavelength optoelectronic devices and spintronic applications. Furthermore, the incorporation of nitrogen to form quaternary GaNAsBi allows lattice matching to GaAs substrates and further band gap reduction. The crystal quality of III-V alloys is highly affected by the growth temperature. A low growth temperature is undesirable as it typically leads to the increase of defect densities and optical quality degradation. However, for the growth of Bi containing alloys, a low growth temperature typically between $270{ }^{\circ} \mathrm{C}$ and $400{ }^{\circ} \mathrm{C}$ is required to avoid $\mathrm{Bi}$ segregation during growth thereby incorporating significant amounts of $\mathrm{Bi}^{3-6}$

It has been reported that increasing $\mathrm{Bi}$ fractions of up to $x=0.045$ leads to an improvement of the optical quality of $\mathrm{GaAs}_{1-\mathrm{x}} \mathrm{Bi}_{\mathrm{x}}{ }^{7}$ Enhanced photoluminescence (PL) in $\mathrm{GaAs}_{1-\mathrm{x}} \mathrm{Bi}_{\mathrm{x}}$ is highly desirable as it may lead to reduced threshold current densities for laser diodes. It was also suggested that the composition dependent PL enhancement was due to localization effects induced by $\mathrm{Bi}$ incorporation similar to the role of indium in wide-gap InGaN and InAlGaN alloys. $^{7,8}$ This is in contrast to $\mathrm{N}$ alloying in GaAs which degrades the optical quality and where localization is associated with defects. However, this result is in contrast with Ref. 9 which observed a decreasing PL intensity with Bi content for $x$ up to 0.026 , in metal-organic vapour phase epitaxy grown samples. To date, the composition dependent PL of $\mathrm{GaAs}_{1-\mathrm{x}} \mathrm{Bi}_{\mathrm{x}}$ has not been verified, and the origin of PL intensity enhancement is still not well understood. In this letter, we report a detailed PL study on the effect of Bi concentra-

a)Electronic mail: j.p.david@sheffield.ac.uk. tion to the optical quality of $\mathrm{GaAs}_{1-\mathrm{x}} \mathrm{Bi}_{\mathrm{x}}$ ( $x$ up to 0.06 ). The reason for PL intensity enhancement also will be addressed.

The samples used in this study were grown using an Omicron molecular beam epitaxy-scanning tunneling microscopy (MBE-STM) system. The semi-insulating GaAs (100) substrate used was cleaved into $11.0 \times 3.5 \mathrm{~mm}^{2}$ pieces to fit into the substrate holder. The cleaning and oxide removal procedures were as described in Ref. 10. A $80 \mathrm{~nm}$ GaAs buffer was grown at $590^{\circ} \mathrm{C}$, followed by $\mathrm{GaAs}_{1-\mathrm{x}} \mathrm{Bi}_{\mathrm{x}}$ layer at a rate of $160 \mathrm{~nm}$ per hour and then capped with 50 $\mathrm{nm}$ of GaAs. Table I summarized the $\mathrm{GaAs}_{1-\mathrm{x}} \mathrm{Bi}_{\mathrm{x}}$ samples studied in this work. The (004) high resolution x-ray diffraction (HRXRD) $\theta / 2 \theta$ scans of the as-grown samples were measured. The epilayer thicknesses and Bi composition were determined by fitting the HRXRD scans using RADS Mercury software, assuming a GaBi lattice constant of 6.324 $\AA$ A. ${ }^{11}$ For the PL measurements, the sample was excited with a continuous-wave $532 \mathrm{~nm}$ wavelength diode-pumped solid state laser. The PL was dispersed using a monochromator and then detected by a liquid nitrogen-cooled germanium detector. A phase-sensitive detection technique was implemented using a lock-in amplifier and a chopper. The spectral response of the PL system (Ge detector, monochromator grating, and associated optics) was determined using a calibrated white light reference source and used to correct the measured PL data.

Figure 1(a) shows the normalized PL data of Set C samples measured at room temperature. The incorporation of $\mathrm{Bi}$

TABLE I. Summary of samples studied in this work.

\begin{tabular}{lccc}
\hline \hline Sample set & $\begin{array}{c}\text { Number } \\
\text { of samples }\end{array}$ & $\begin{array}{c}\text { GaAsBi } \\
\text { thickness (nm) }\end{array}$ & $\begin{array}{c}\text { GaAsBi growth } \\
\text { temperature }\left({ }^{\circ} \mathrm{C}\right)\end{array}$ \\
\hline A & 3 & 100 & 420 \\
B & 3 & 50 & 400 \\
C & 4 & 25 & 400 \\
\hline \hline
\end{tabular}



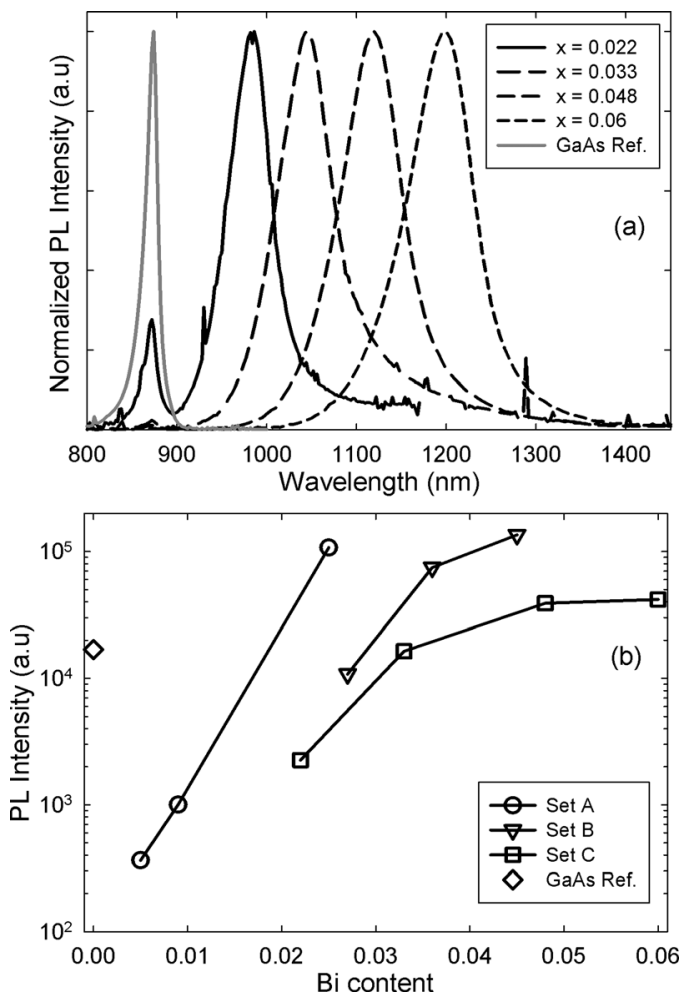

FIG. 1. (a) Normalised room temperature PL spectra for Set C samples (b) $\mathrm{PL}$ intensity versus Bi content at room temperature with $P_{\mathrm{e}}=320 \mathrm{Wcm}^{-2}$.

reduces the band gap of $\mathrm{GaAs}$ and the highest $\mathrm{Bi}$ content sample, $\mathrm{GaAs}_{0.94} \mathrm{Bi}_{0.06}$ has a peak wavelength of $1.2 \mu \mathrm{m}$. The band gap reduction is mainly due to the increase of valence band maximum due to the valence band anti-crossing interaction. $^{12}$ However, the full-width-at-half-maximum (FWHM) lies between 74 and $84 \mathrm{meV}$, which is significantly larger than the FWHM of GaAs (26 meV). Figure 1(b) shows the PL intensity comparison as a function of Bi content with excitation power, $P_{e}=320 \mathrm{Wcm}^{-2}$. The PL intensity of $\mathrm{GaAs}_{0.94} \mathrm{Bi}_{0.06}$ sample is approximately 100 times greater than the thicker $\mathrm{GaAs}_{0.995} \mathrm{Bi}_{0.005}$ sample. Interestingly, the PL intensity of $\mathrm{GaAs}_{0.94} \mathrm{Bi}_{0.06}$ is also twice compared to the PL intensity of a high quality $3 \mu \mathrm{m}$ thick GaAs reference sample (grown by standard V90 $\mathrm{MBE}$ at $580^{\circ} \mathrm{C}$ ). Even though the GaAs sample is a different structure, the comparison still gives useful information about the crystal quality of the $\mathrm{GaAs}_{1-\mathrm{x}} \mathrm{Bi}_{\mathrm{x}}$ samples. The PL intensity increases rapidly at low $\mathrm{Bi}$ content but starts to saturate at high Bi composition. The composition dependent PL enhancement may be due to (i) higher Bi content reduces the defect density, (ii) greater carrier confinement due to larger GaAs/GaAsBi band offset for high $\mathrm{Bi}$ content samples, or (iii) higher Bi content increases the localisation effects near the valence band maximum, efficiently trapping holes and making excitons less sensitive to non-radiative recombination centers.

In order to verify the presence of localization effects in the samples, temperature dependent PL measurements were carried out. The measurements used $P_{e}=53 \mathrm{Wcm}^{-2}$, power density that avoids saturating the localized states while ensuring measurable PL signals from all samples at room temperature. The data of PL peak energy against temperature for Set C samples are shown in Figure 2(a). All samples
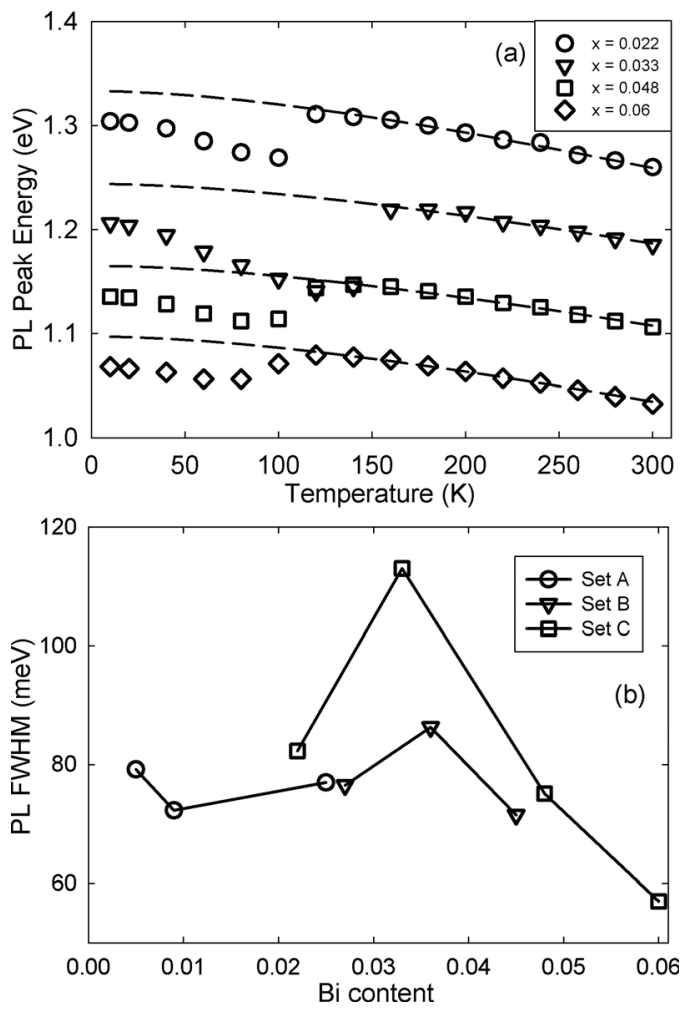

FIG. 2. (a) Temperature dependent of PL peak energy of Set $\mathrm{C}$ samples and (b) FWHM measured at $10 \mathrm{~K}$ when excited with $P_{\mathrm{e}}=53 \mathrm{Wcm}^{-2}$. The dashed lines are fitting using Varshni equation.

showed the S-shape behavior which is a well-known signature of localization effects. However, the localization energy varies from sample to sample. The data were fitted using the Varshni equation, ${ }^{13}$

$$
E_{g}(T)=E_{o}-\alpha T^{2} /(T+\beta),
$$

where $E_{o}$ is the $0 \mathrm{~K}$ band gap and $\alpha$ and $\beta$ are fitting parameters and these are summarized in Table II. The localization energy for a particular temperature is defined as $E_{l o c}$ $(T)=E_{g}(T)-E_{P L}(T){ }^{14}$ The value of the maximum localization energy, $E_{\text {maxloc }}$, is also included in Table II. As $x$ increases from 0.022 to $0.033, E_{\text {maxloc }}$ initially increases from 51 to $90 \mathrm{meV}$ and then decreases to 46 and $36 \mathrm{meV}$ for $x=0.048$ and 0.06 , respectively. From Figure 2(b), the $10 \mathrm{~K}$ FWHM of Set $\mathrm{C}$ samples also shows a maximum at $x=0.033$, consistent with the trend observed for $E_{\text {maxloc }}$. This observation is expected as the $10 \mathrm{~K}$ linewidth is mainly affected by alloy fluctuations and Bi clustering, thus indicative of the relative localization strength. However, for Set A and B samples, the linewidth is approximately constant with an average value of $77 \mathrm{meV}$. This means that the localization energy is unchanged for both sets of samples. Data in

TABLE II. Varshni fitting parameters and $E_{\text {maxloc }}$ for Set C samples.

\begin{tabular}{lcccc}
\hline \hline Bi content & $E_{\text {maxloc }}(\mathrm{meV})$ & $E_{o}(\mathrm{eV})$ & $\alpha(\mathrm{meV} / \mathrm{K})$ & $\beta(\mathrm{K})$ \\
\hline 0.022 & 51 & 1.333 & 0.45 & 250 \\
0.033 & 90 & 1.244 & 0.37 & 280 \\
0.048 & 46 & 1.165 & 0.37 & 280 \\
0.060 & 36 & 1.097 & 0.39 & 260 \\
\hline \hline
\end{tabular}




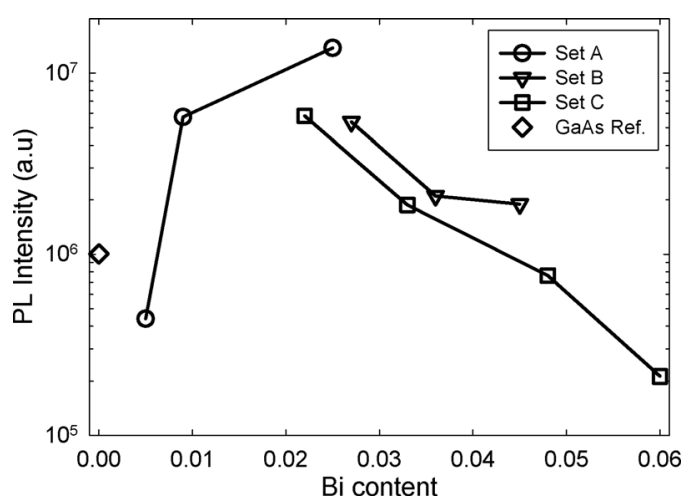

FIG. 3. PL intensity measured at $10 \mathrm{~K}$ with $P_{\mathrm{e}}=53 \mathrm{Wcm}^{-2}$.

Figure 2 do not show an increase in localization energy and FWHM with increasing $x$ for the range studied. Hence, localization effects are unlikely to be the main mechanism for the increased PL shown in Figure 1(b).

Figure 3 shows the $10 \mathrm{~K}$ PL intensity plotted as a function of Bi concentration. For Set A samples, the PL intensity increases with increasing Bi content but decreases for Sets B and $\mathrm{C}$. The results are in contrast with the room temperature PL intensity trend observed in Figure 1(b). The results suggest that the incorporation of $\mathrm{Bi}$ in GaAs of up to $\sim 0.025$ improves the material quality. It is well known that growing GaAs at significantly lower than the optimal growth temperature $\left(580^{\circ} \mathrm{C}\right)$ induces many defects such as As-related defects (As-interstitial ${ }^{15}$ and As-antisite, $\mathrm{As}_{\mathrm{Ga}}{ }^{16}$ ) and $\mathrm{Ga}$ vacancies. ${ }^{17}$ Incorporating Bi during low temperature growth enhances surface migration, thus reducing the density of Ga and/or As-related defects. ${ }^{18}$ Based on deep level transient spectroscopy (DLTS) study reported recently, the trap concentrations in $\mathrm{GaAs}_{0.988} \mathrm{Bi}_{0.012}$ is $\sim 10$ times lower than $\mathrm{GaAs}$, when both are grown at $370{ }^{\circ} \mathrm{C} .{ }^{18}$ However, Bi incorporation also introduces Bi-related defects such as Bi-antisite, $\mathrm{Bi}_{\mathrm{Ga}} \cdot{ }^{19}$ For $x>0.025$, the Bi-related defects started to become significant, thus degrade the optical quality of $\mathrm{GaAs}_{1-\mathrm{x}} \mathrm{Bi}_{\mathrm{x}}$. This explains the PL intensity reduction for set $\mathrm{B}$ and $\mathrm{C}$ measured at $10 \mathrm{~K}$.

The room temperature PL intensity enhancement observed in Figure 1(b) is the result of more efficient carrier confinement (and higher quality for $x<0.025$ ) due to a larger band gap offset (between GaAs and $\mathrm{GaAs}_{1-\mathrm{x}} \mathrm{Bi}_{\mathrm{x}}$ ) with increasing $\mathrm{Bi}$ content. The PL enhancement slowly saturates when the valence band offset becomes large enough to avoid carrier escape. It is expected that the room temperature PL intensity will start to decrease for $x>0.06$ due to material quality degradation. In Ref. 7, the room temperature PL intensity increases only up to 0.045 (in this work up to 0.06 ) probably due to higher density of Bi-related defects as a result of lower growth temperature $\left(270-300^{\circ} \mathrm{C}\right)$. It is important to note that even though the crystal quality gradually degrades for $x>0.025$, the PL intensity of $\mathrm{GaAs}_{0.94} \mathrm{Bi}_{0.06}$ (at room temperature and $10 \mathrm{~K}$ ) is still comparable to the PL intensity of the high quality GaAs reference sample.

In summary, for $\mathrm{GaAs}_{1-\mathrm{x}} \mathrm{Bi}_{\mathrm{x}}$ samples grown by MBE, the optical quality is highly dependent on the $\mathrm{Bi}$ concentration. The incorporation of $\mathrm{Bi}$ in GaAs reduces the density of $\mathrm{Ga}$ and/or As-related defects as well as introducing Birelated defects. For dilute amount of $\mathrm{Bi}(x<0.025)$, the crystal quality improves but further increase of Bi causes degradation to the material quality probably due to significant amount of Bi-related defects. However, the room temperature PL continues to increase up to $x=0.06$ due to greater band-gap offset between $\mathrm{GaAs}$ and $\mathrm{GaAs}_{1-\mathrm{x}} \mathrm{Bi}_{\mathrm{x}}$ layer. No clear correlation was found that relates the localization effect to the room temperature PL intensity enhancement.

The authors would like to thank Professor M. S. Skolnick for helpful discussions. This work was supported by the UK Technology Strategy Board "Extended Temperature Optoelectronics II," the UK EPSRC-University of Sheffield Doctoral Fellowship (F. Bastiman), and the Royal Society (University Research Fellowship for J. S. Ng).

${ }^{1}$ S. Francoeur, M. J. Seong, A. Mascarenhas, S. Tixier, M. Adamcyk, and T. Tiedje, Appl. Phys. Lett. 82, 3874 (2003).

${ }^{2}$ W. Huang, K. Oe, G. Feng, and M. Yoshimoto, J. Appl. Phys. 98, 053505 (2005).

${ }^{3}$ S. Tixier, M. Adamcyk, T. Tiedje, S. Francoeur, A. Mascarenhas, P. Wei, and F. Schiettekatte, Appl. Phys. Lett. 82, 2245 (2003).

${ }^{4}$ K. Bertulis, A. Krotkus, G. Aleksejenko, V. Pacebutas, R. Adomavicius, G. Molis, and S. Marcinkevicius, Appl. Phys. Lett. 88, 201112 (2006).

${ }^{5}$ M. Henini, J. Ibanez, M. Schmidbauer, M. Shafi, S. V. Novikov, L. Turyanska, S. I. Molina, D. L. Sales, M. F. Chisholm, and J. Misiewicz, Appl. Phys. Lett. 91, 251909 (2007).

${ }^{6}$ X. Lu, D. A. Beaton, R. B. Lewis, T. Tiedje, and M. B. Whitwick, Appl. Phys. Lett. 92, 192110 (2008).

${ }^{7}$ X. Lu, D. A. Beaton, R. B. Lewis, T. Tiedje, and Y. Zhang, Appl. Phys. Lett. 95, 041903 (2009).

${ }^{8}$ S. F. Chichibu, A. Uedono, T. Onuma, B. A. Haskell, A. Chakraborty, T. Koyama, P. T. Fini, S. Keller, S. P. DenBaars, J. S. Speck, U. K. Mishra, S. Nakamura, S. Yamaguchi, S. Kamiyama, H. Amano, I. Akasaki, J. Han, and T. Sota, Nat. Mater. 5, 810 (2006).

${ }^{9}$ K. Oe, Jpn. J. Appl. Phys. 41, 2801 (2002).

${ }^{10}$ A. R. Mohmad, F. Bastiman, J. S. Ng, S. J. Sweeney, and J. P. R. David, Appl. Phys. Lett. 98, 122107 (2011).

${ }^{11}$ A. Janotti, S. H. Wei, and S. B. Zhang, Phys. Rev. B 65, 1152031 (2002).

${ }^{12}$ K. Alberi, O. D. Dubon, W. Walukiewicz, K. M. Yu, K. Bertulis, and A. Krotkus, Appl. Phys. Lett. 91, 051909 (2007).

${ }^{13}$ Y. P. Varshni, Physica (Amsterdam) 34, 149 (1967).

${ }^{14}$ M. A. Pinault and E. Tournie, Appl. Phys. Lett. 78, 1562 (2001).

${ }^{15}$ N. Hozhabri, S. H. Lee, and K. Alavi, Appl. Phys. Lett. 66, 2546 (1995).

${ }^{16}$ M. Kaminska, Z. Liliental-Weber, E. R. Weber, T. George, J. B. Kortright, F. W. Smith, B. Y. Tsaur, and A. R. Calawa, Appl. Phys. Lett. 54, 1881 (1989).

${ }^{17}$ J. Gebauer, R. Krause-Rehberg, S. Eichler, M. Luysberg, H. Sohn, and E. R. Weber, Appl. Phys. Lett. 71, 638 (1997).

${ }^{18}$ T. Fuyuki, S. Kashiyama, Y. Tominaga, K. Oe, and M. Yoshimoto, 38th International Symposium on Compound Semiconductors, Berlin, 22-26 May 2011.

${ }^{19}$ M. Kunzer, W. Jost, U. Kaufmann, H. M. Hobgood, and R. N. Thomas, Phys. Rev. B 48, 4437 (1993). 